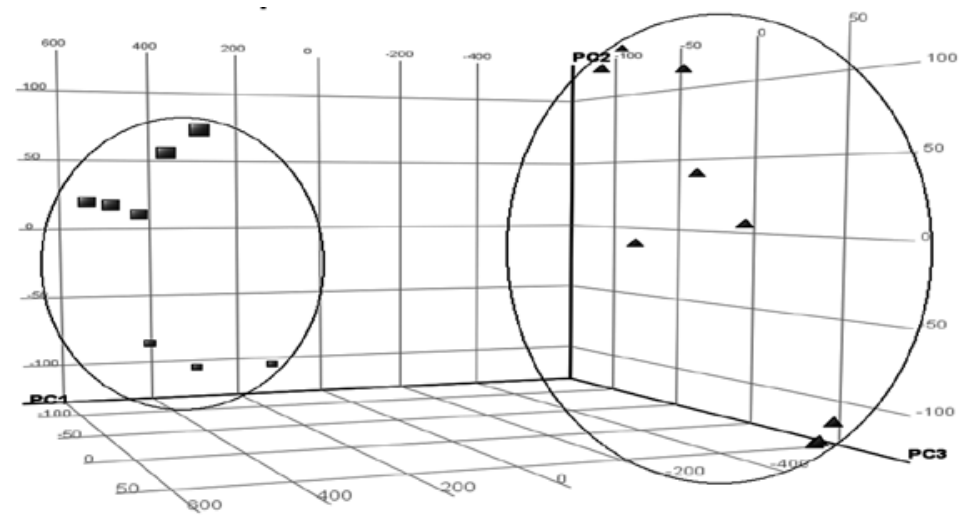

Shape by geographical distribution

- Cairns

$\Delta$ Townsville the six isolates were grown on agar plates and extracted with ethyl acetate. Ultra-high Performance Liquid Chromatography hyphenated to Quadrupole Time of Flight Mass Spectrometry (UPLC-QTOFMS) was used to obtain the secondary metabolomic profiles and chemometric analysis was used for data processing. 3-D PCA scores plot based on the HPLC-QTOF-MS profiles are shown below:
A significant level of diversity was evident within the secondary metabolome of $S$. arenicola strains isolated from two distinct regions of the GBR.

References: (1) Maldonado LA, et al., Int J Syst Microbiol 55, 1759-1766 (2005). (2) Jensen PR, et al., Appl Environ Microbiol 73 (4): 1146-1152 (2007).

\title{
ESSENTIAL OIL COMPOSITION OF ANGELICA ARCHANGELICA ROOT FROM NORTHERN FINLAND
}

\section{(C) Holm Y. ${ }^{1}$, Galambosi B. ${ }^{2}$, Heikkinen J. ${ }^{3}$, Galambosi Zs. ${ }^{2}$, Hiltunen R. ${ }^{1}$}

${ }^{1}$ Division of Pharmaceutical Biology, Faculty of Pharmacy, University of Helsinki, Finland ${ }^{2}$ MTT Agrifood Research Finland, Mikkeli, Finland

${ }^{3}$ Lapland Vocational College, Rovaniemi, Finland

The genus Angelica is native to temperate and subarctic regions of the northern hemisphere and the samples for this study were collected in seven different locations in northern Finland and cultivated at MTT Agrifood Research in Mikkeli, south eastern Finland in 2011. We had ten samples of Angelica archangelica ssp. angelica $\mathrm{L}$. and one sample of $A$. archangelica ssp. littoralis (Fr.) Thell. The aim was to compare the oil composition of Finnish $A$. archangelica with samples of $A$. archangelica from Greenland and the Faroe Islands cultivated in Sweden, which we investigated a few years ago (1). The root samples were chopped with a cutter and hydrodistilled in a Karlsruher-Stahl apparatus for two hours. The oil yield varied between 0.26 and $1.16 \%$. The essential oils were analysed by GC-MS on both a polar (Stabilwax) and a non-polar (RTX-1) column. The major part of the oil consisted of monoterpenes (70-83\%), with alfa- pinene, alfa- and beta-phellandrene as main components. In addition decanolides (2-15\%) and coumarins, i. e. osthol, were found in small percentages. Comparison with the Swedish samples revealed that they had a lower content of monoterpenes (60-70\%) and a higher content of decanolides (7-20\%). In conclusion, it can be stated that $A$. archangelica root oil consists mostly of monoterpenes, but the decanolides are important for the flavour of the oil (2).

References: (1) Holm Y, Solberg S, Hiltunen R. Variation in Angelica archangelica root essential oils. Planta Med 75, 929, 2009. (2) Lopes D, Strohl H, Kolodziejczyk P. 14-Methylpentadecano-15-lactone (muscolide): A new macrocyclic lactone from the oil of Angelica archangelica L. Chemistry \& Biodiversity 1 , 1880-1887, 2004. 